\title{
Endogenous reward mechanisms and their importance in stress reduction, exercise and the brain
}

Tobias Esch ${ }^{1,2}$, George B. Stefano ${ }^{2}$

${ }^{1}$ Division of Integrative Health Promotion, Coburg University of Applied Sciences, Coburg, Germany

${ }^{2}$ Neuroscience Research Institute, State University of New York, Old Westbury, USA

Submitted: 17 May 2010

Accepted: 13 June 2010

Arch Med Sci 2010; 6, 3: 447-455

DOI: 10.5114 /aoms.2010.14269

Copyright $\odot 2010$ Termedia \& Banach

\begin{abstract}
Stress can facilitate disease processes and causes strain on the health care budgets. It is responsible or involved in many human ailments of our time, such as cardiovascular illnesses, particularly related to the psychosocial stressors of daily life, including work. Besides pharmacological or clinical medical treatment options, behavioral stress reduction is much-needed. These latter approaches rely on an endogenous healing potential via life-style modification. Hence, research has suggested different ways and approaches to self-treat stress or buffer against stressors and their impacts. These self-care-centred approaches are sometimes referred to as mind-body medicine or multi-factorial stress management strategies. They consist of various cognitive behavioral techniques, as well as relaxation exercises and nutritional counselling. However, a critical and consistent element of modern effective stress reduction strategies are exercise practices. With regard to underlying neurobiological mechanisms of stress relief, reward and motivation circuitries that are imbedded in the limbic regions of the brain are responsible for the autoregulatory and endogenous processing of stress. Exercise techniques clearly have an impact upon these systems. Thereby, physical activities have a potential to increase mood, i.e., decrease psychological distress by pleasure induction. For doing so, neurobiological signalling molecules such as endogenous morphine and coupled nitric oxide pathways get activated and finely tuned. Evolutionarily, the various activities and autoregulatory pathways are linked together, which can also be demonstrated by the fact that dopamine is endogenously converted into morphine which itself leads to enhanced nitric oxide release by activation of constitutive nitric oxide synthase enzymes. These molecules and mechanisms are clearly stress-reducing.
\end{abstract}

Key words: nitric oxide, morphine, dopamine, pleasure, motivation, neurobiology, autoregulation, health, running, mind-body medicine.

\section{Introduction}

Stress is an eminent challenge nowadays. This seems to be especially true for modern service-oriented societies, where the stress phenomenon and its impact on individual health, work force, environments, as well as the national economies and business profits, even overall "happiness scores" (i.e., the well-being of individuals and the society on a larger scale), is evident [1-6]. In fact, not only seems stress to be increasing in reference to medical operating figures and determinants, but has particularly

\author{
Corresponding author: \\ Prof. Dr. Tobias Esch \\ Coburg University \\ of Applied Sciences \\ Friedrich-Streib-Str. 2 \\ D-96450 Coburg, Germany \\ Phone: +49 9561317372 \\ Fax: +49 9561317326 \\ E-mail: esch@hs-coburg.de
}


penetrated into the daily lives of even the young and otherwise healthy, thus causing potential threat [1-3]. In the course of this increasing stress proliferation and overflow, as it seems, the remaining "islands of stillness" and daily life recoveries seem to advance backward, and people search for wellness and recreation at all kinds of places, e.g., short-time or "wellness in a rush" [4-6].

One of the major problems related to the given description of stress and its significance in our times is that most of the stress that we encounter or perceive seems not to be an actual physical or external life threat, but instead, to some extent, "self-made", that is, produced in our heads and thoughts, i.e., cognitively, or posed to each other by psychosocial or chronic conflict $[1,4]$. Thus, data for the work environments, for example, demonstrate that the psychosocial stress is generally increasing, leading to accelerating absenteeism and/or "presentism" rates (i.e., going to work but not utilizing the full individual potential), all together "unhealthy" for the individuals, the companies, and the societies $[1,3]$.

No doubt, circumstances and external forces, e.g., financial or economical crisis, are responsible for a lot of threat and stress that people have to face, accessory to demographic or socioeconomic factors, noise, pollution, etc., however, individual behavior and resources are of importance for stress as well, as for stress resilience and relief [4-6]. Thus, besides influencing external and political or environmental factors, which is a predominant and important task for health care, economical and particularly educational policies, the endogenous or self-regulatory potentials to individually reduce or buffer against stress, when stress is simply there (and it is!) or yet has to be faced, is also of significance and should not be neglected.

We have an endogenous and salutogenic potential for stress resilience [5], and to increase or use this potential, it is sometimes helpful to seek professional advice and support, since for many individuals the autoregulation of stress has gotten out of the focus during their life spans and routines, that is, trapped or blocked [4, 6]. Hence, modern stress management approaches help to activate this potential and re-connect to it, and exercise is one of their key elements. Recent neurobiological research explains some of the observations and scientific background.

\section{Stress}

Stress describes the effects of psychosocial and environmental factors on physical or mental well-being $[6,7]$. Stressors and related stressreactions are often distinguished [4, 6]. Further, stress implies a challenge (stimulus) that requires behavioral, psychological, and physiological changes (adaptations) to be successfully met, therefore using a state of hyperarousal for the initiation of necessary counteracting reactions $[6,7]$. This state of hyperarousal involves physiological mechanisms that are known as the stress/emergency response or fight-or-flight response, a set of physiological changes that occur in stressful situations and that prepare the stressed organism either to fight or to flee. The state of alertness had first been described by Walter Cannon - almost 100 years ago [8, 9].

Stress has an impact upon the immune, circulatory, and nervous systems [6]. Therefore, stress may affect immunological [10], cardiovascular [11], and neurodegenerative diseases/mental disorders [12], and this may include both positive and negative aspects [6,10-12]. Thus, stress can either exert ameliorating or deleterious effects, depending on a multitude of factors (e.g., individual, endogenous, or exogenous elements) $[4,6]$. However, clinically, and with reference to modern medical settings, negative influences of stress upon health and disease processes seem to dominate $[2,3,6,10-12]$. This may especially be true in the 'Western World', where stress-related health issues almost seem to have an increasingly epidemic character [1-7].

Additionally, the concept of 'allostasis' has been introduced into the field of stress medicine/research by Sterling and Eyer [13], refined by McEwen [14]. Thereby, allostasis, literally meaning "maintaining stability (or 'homeostasis') through change" [10, 13, 14], describes the capacity to adapt or to constantly change, i.e., modify, physiological parameters in order to adjust to ever shifting environmental conditions [4, 6, 7, 12]. Hence, we can also speak alternatively about maintaining a 'state of dynamic balance' [10-12]. Moreover, 'allostatic load' refers to the wear and tear that the body experiences due to repeated cycles of allostasis, i.e., allostatic stress responses, as well as the inefficient turning-on or shutting-off of these activated responses $[6,10,14]$.

When the brain perceives/senses an experience or stimulus as stressful, physiologic and behavioral responses (i.e., stress responses) are initiated, leading to allostasis and adaptation (i.e., adaptive/allostatic stress responses) [7, 14]. The goal is to keep balance, self-organize, and maintain autonomy under challenge, and ultimately to survive [6]. As a result of this ongoing adaptation, over time, allostatic load can accumulate, and the overexposure to neural, endocrine, and immune stress mediators can have adverse effects on various organ systems, leading to the onset or progression of diseases [6, 10-12, 14].

Today, two molecules that play a major role in the stress response are thoroughly examined and their functions well-known. Each molecule represents one arm of the response (the hypo- 
thalamic-pituitary-adrenal (HPA) axis and the sympathoadrenal medullary (SAM) system [15]). They are cortisol and norepinephrine (NE)/epinephrine $[6,8,9,15]$. More recently, other molecules involved have been detected, e.g., melatonin or anandamide, and the connection of endorphins and nitric oxide (NO) with the stress response has additionally been proposed [5-7, 16-19].

\section{Endogenous reward}

Research has identified a biological mechanism mediating behavior motivated by events commonly associated with pleasure. This mechanism is called reward. It is usually governing normal behavior through pleasurable experiences [20, 21]. Pleasure, however, describes a 'state or feeling of happiness or satisfaction resulting from an experience that one enjoys' [20]. Pleasure is a subjective phenomenon, i.e., subjective quality. Hence, an intimate association between reward and pleasure exists [20, 21]. In neurobiology, pleasure is a competence or function of the reward and motivation circuitries that are imbedded in the central nervous system (CNS). Anatomically, these reward pathways are particularly linked to the brain's limbic system. The underlying physiology, however, is complex and morphological correlates are still a matter of thorough research.

Motivation can be divided into two categories, i.e., appetitive and aversive motivation. Appetitive motivation concerns behavior directed towards goals that are normally associated with positive hedonic, i.e., pleasurable, processes (food, recreational drugs, sex etc.). In contrast, aversive motivation involves getting away from hedonically unpleasant conditions [21, 22]. Consequently, two fundamental forces rule motivation: pleasure and pain. It has been suggested that pleasure may be associated with beneception, events that facilitate survival and thus benefit the organism or species from an evolutionary biology perspective [23]. Pain, on the other hand, is associated with nociception. This latter term basically describes conditions that may have undesirable biological consequences for an organism [21, 23]. However, the illustrated division of pleasure and pain in reference to their possible biological functions and outcome should not lead to an incorrect understanding, since both conditions - in specific situations - may have the capacity to serve survival likewise. Thus, pain and pleasure potentially merge into another. With regard to specialized brain compartments involved in motivational processes, the physiological substrate for appetitive or aversive motivation (as for reward and avoidance) primarily lies within the limbic system [20, 24].

The common idea that the limbic system is solely concerned with feelings and emotion is at best a half-truth, but there certainly exists a connection which is relevant to the pleasure phenomenon [20]. Yet, the limbic system is made up of the limbic lobe and certain additional structures. The limbic lobe surrounds the corpus callosum and consists of the cingulate gyrus and the parahippocampal gyrus. The hippocampus, which is in the floor of the temporal horn of the lateral ventricle and is closely linked to memory processing, is also included in the limbic lobe [20, 25]. Additional structures incorporated in the limbic system are the dentate gyrus, amygdala, hypothalamus (mammillary bodies), septal area (in the basal forebrain) and thalamus (anterior and some other nuclei). Functionally, the 'hippocampal formation' consists of the hippocampus, the dentate gyrus and most of the parahippocampal gyrus [20]. Neurobiologists have long known that the euphoria induced by drugs of abuse, sex or other things we enjoy arises because all these factors ultimately boost the activity of the brain's pleasure and reward systems. These are made up of complex circuits of nerve cells or neurons that evolved to make us feel flush after eating or sex, i.e., things we need to do to survive and pass along our genes [26, 27]. Reward pathways are evolutionarily ancient, like limbic structures. Limbic and reward systems share common mechanisms and morphological structures. In fact, integral CNS components involved in reward and motivational processes are of limbic origin [20, 25]. For example, prefrontal or orbitofrontal cortices, cingulate gyrus, amygdala, hippocampus and nucleus accumbens participate in the reward physiology [20]. Thus, pleasure, limbic system and reward mechanisms are functionally and biologically interconnected. Memories of the pleasure of wellness, i.e., 'remembered wellness', are accessible to this circuitry through hippocampal mechanisms [20, 25]. Further, belief affects mesocortical-mesolimbic appraisal of a pleasurable experience, leaving one, for example, well and relaxed.

A crucial component of CNS reward and motivation circuitries are nerve cells that originate in the ventral tegmental area (VTA), near the base of the brain. These cells send projections to target regions in the frontal brain, most notably to a structure deep beneath the frontal cortex, i.e., nucleus accumbens $[26,27]$. The essential neurotransmitter of this connection is dopamine. Clearly, the VTA or mesolimbic dopamine system represents a rather old but very effective part of motivational physiology and behavior. However, in humans, the reward circuit is more complex, and it is integrated with several other brain regions that serve to enrich an experience with emotion (as an example) and direct the individual's response or behavior toward rewarding stimuli, including food, 
sex and social interaction [20]. The amygdala, for instance, is a special part of limbic and reward systems that is closely related to emotion (especially fear) and has many post-synaptic receptors for which $\gamma$-aminobutyric acid (GABA) is an inhibitory neurotransmitter [20, 25]. Diazepam and other anxiolytics mimic the action of GABA at this site. Researchers have hypothesized that pleasurable experiences like various complementary medical treatments, e.g., acupuncture or meditation, may exert calming effects via release of GABA in the amygdala and other limbic areas [5, 19, 20, 25]. This speculative aspect may be supported by recent findings that link endogenous morphine production to limbic structures and complementary or alternative medicine (CAM) [5, 16-20, 25]. In fact, morphine is endogenously synthesized via dopamine, and dopamine is another key transmitter found in the amygdala [5, 17, 20, 28]. Thus, on the neurochemical level, pleasure may involve substances that possess calming and anxiolytic capacities, thereby facilitating feelings of well-being and relaxation.

The amygdala also helps to assess whether an experience is pleasurable or aversive (and whether it should be repeated or avoided) and further helps to forge connections between an experience and other cues [26, 27]. Meanwhile, the hippocampus participates in recording memories of an experience, including where, when and with whom it occurred [20]. The frontal cortex, however, coordinates and processes all this information and consequently determines the ultimate behavior. Finally, the VTA-accumbens pathway acts as a measuring tool and regulator of reward: it 'tells' the other brain centers how rewarding an activity is [20]. The more rewarding an activity is deemed, the more likely the individual is to remember it well and repeat it.

With regard to frequent neuronal reward 'tracks' within the CNS, activation of the medial forebrain bundle (MFB), as it courses through the lateral hypothalamus to the ventral tegmentum, has been shown to produce robust rewarding effects. Dopamine is substantially involved. Electrophysiological and neurochemical techniques revealed: CNS stimulation may activate a descending component of the MFB which is synaptically coupled at the ventral tegmentum to the ascending mesolimbic dopamine system, i.e., nucleus accumbens [20, 21, 29]. Pleasure-inducing electrical stimulation thus involves a circuitous reward pathway, first activating a descending MFB component and then the ascending mesolimbic dopamine pathway. Clearly, we can speak of brain's reward and motivation circuitries.

Taken together, the brain possesses specialized pathways that mediate pleasure, reward and motivation. Psychomotor stimulants and opiates, similar to experimental electrical stimulation, activate this reward system by their pharmacological actions in the VTA and nucleus accumbens [20, 26, 27, 29]. Ventral tegmental activation, however, as well as other essential CNS reward features involve dopamine signalling. Other neurotransmitters (e.g., GABA, glutamate, serotonin, stress hormones) may play a critical role too [5, 20]. In addition, endogenous morphine/opioid peptide production may be of importance [5]. Natural rewards like food and sex in accordance with other substances, such as caffeine, ethanol, nicotine etc., may also activate brain's reward and motivation circuitries [29]. However, the primary access to this system, at least from a biological perspective, seems to happen via endogenous or self-regulative behaviors and activities [5].

\section{Stress reduction, exercise, brain}

Exercise can reduce obesity, depression, and/or stress, to only name a few inter-correlations of substantial medical significance (e.g., see [5, 6, 30]). Physical activity, particularly aerobic exercise, can improve a number of aspects of cognition and bodily performance [31-33]. Dynamic physical activities, but not static tasks or sedentary lifestyles, lead to a marked increase of regional cerebral blood flow [34-37]. Lack of physical activity, especially among children, is a major cause for obesity and the early onset of many diseases and consecutive aggravation in adult life, e.g., cardiovascular or metabolic [31, 38]. However, there is substantial evidence mounting up that suggests exercise not only to be highly recommendable for keeping and enhancing physical health, but also to improve academic and mental performance, i.e., 'running makes smart' [31-33, 39]. We suggest that this effect may, in parts, be attributed to the endogenous stress reduction and protection potential, and a related neurobiological involvement of limbic portions of the brain as well as the underlying molecular pathways, already displayed above.

Physical activity is a lifestyle factor that leads to increased health and stress hardiness throughout life [5, 31, 32, 35-37, 40]. It's good to start early and not give up this practice while growing up. However, even the physical and mental decline of the elderly is not completely inherent or predestined, only reliant on genes or 'fate', but instead, partly reversible. Yet, these age-dependent processes can be delayed, some prevented, compensated, or even reversed, 'just' by starting to get active again during old age [32, 37, 39-41]. The critical aspect for this potential is the life-long ability of the brain to adjust and adapt, actually on the molecular level, and this capacity can be trained, i.e., neuroplasticity $[5,41]$. 
A very capable training stimulus therefore seems to be mild and manageable mental stimulation or mild aerobic exercise [5, 35, 37, 39-41]. This neuroplastic effect of exercise therapy also works with stroke patients, demonstrating it as an effective life or survival strategy [42, 43].

Appropriate lifestyle interventions or purposeful activities can positively influence the reserve capacity of aging humans and the aging process itself, particularly with regard to physiological development, cognitive performance, longevity, as well as the onset and course of chronic diseases, including stress regulation (Figure 1). Cognitive development and state are plastic, i.e., flexible, facilitated by positive or pleasurable behaviors (see above) and activities, cognitive as well as physical [32]. Clearly, body and mind are interconnected [5, 37, 44-49]. For using this capacity, it is important to train cognitive and physical flexibility likewise, which seems to be true for stress management (SM) as well [6, 33, 44, 50]. However, the effect of physical activity on cognitive function, particularly but not exclusively in the elderly, turns up to be eminently impressive [5].

Physical exercise improves learning. Obviously, this effect is based upon the enhancement of neurogenesis in the hippocampus through bodily activity, a brain area critical for learning and memory, particularly with regard to the declarative long-term memory, and it is part of the limbic brain [12, 39, 51, 52]. Clearly, there exists a positive neurobiological correlation between running and neurogenesis [51]. Interestingly, the hippocampus is also extremely sensitive to stress, since psychosocial or mental stress, in particular, tend to deteriorate neurons in the hippocampus, leading to accelerated neurodegeneration and possibly dementia [12, 53, 54]. As with stress, aging causes hippocampal decline [39]. This negative effect of stress - and aging - is potentially counteracted by exercise. In fact, stress has a direct impact upon the production/release of brain-derived neurotrophic factor (BDNF), which itself is strongly linked to the serotonin system and plays an essential role in mood and memory processes [50, 52]. More precisely, stress decreases BDNF levels, particularly in the prefrontal cortex where the working memory is located [50]. Moreover, brain tryptophan levels and serotonin metabolism correlate positively with BDNF in both prefrontal cortex and hippocampus, again highlighting the close interconnection and commonality between the underlying signalling systems $[5,50]$. This appears to be also true for the stress-reward-memory-opioid/opiate connection (e.g., $[5,12,53])$, although a rather complex matter and not fully understood yet.

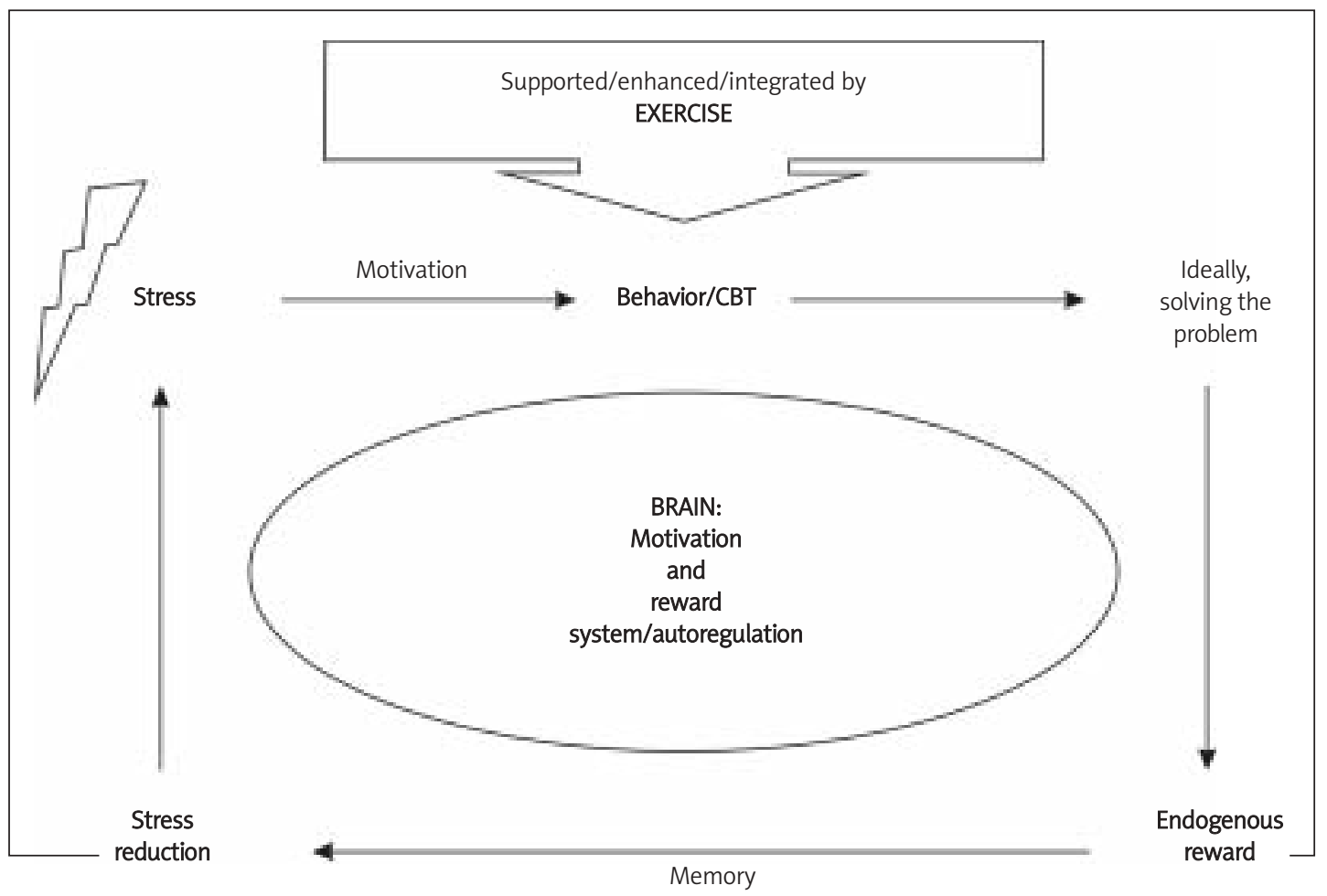

Figure 1. Stress regulation and reward - exercise influences. Further explanations and references see text CBT - cognitive behavioral therapy 
Stress alters memory performance, and morphine interacts with this phenomenon, be it as a primary target of stress-related memory alteration or, supposedly, as a secondary back-up player, i.e., autoregulation, following a stress-related 'narrowing' of the memory focus and attention concentration with the putative goal of deleting every memory input that, in the moment of fight or flight (acute stress), would negatively interfere and not serve the hoped for positive outcome for the fighting individual: while stressed, new or other working memory contents are hardly learned, that is, acquired and consolidated [12, 52, 53]. However, it seems to be biologically essential to keep the 'idea' of a successful strategy that helped to fight the stressor in mind, and somehow accessible afterwards, so that after the fight is over, this successful strategy can become endogenously evaluated and, if positive, memorized and learned (Figure 1). Morphine seems to play a critical role in this process, for example, as a recovery or secondary back-up molecule $[5,53]$. Morphine is critical for learning, and since it is chemically linked to dopamine, i.e., derived from it, it links the pleasure of an actual event (e.g., the felt 'challenge', which is neurobiologically related to dopamine signalling) with the positive memorization of it and positive future expectance (i.e., placebo response) and, additionally, induces NO release - thus, it is not surprising to find morphine and NO in memory processing [5, 20, 53, 55].

As stated above, exercise enhances learning, function and neurogenesis in the hippocampus and the prefrontal cortex, e.g., via BDNF, and this cascade is negatively influenced by stress $[40,50$ 52, 54]. More precisely, stress decreases BDNF, exercise increases it, given as an example. In fact, stress and physical activity are neurobiological counter-players [5, 6], and stress reduction through exercise not only improves memory functions, hippocampal neurogenesis and BDNF levels, but also mood, quality of life, and overall well-being [5, $12,52,56]$. However, this seems to apply only to moderate exercise, since prolonged and strenuous physical activity, for example, can itself cause stress and proinflammation [57-61]. Besides the prefrontal or orbitofrontal and hippocampal areas of the brain, there are other regions that also seem to be of importance in the neurobiological stress-SMexercise-cognition relationship, e.g., temporal cortex, bilateral insula and parainsular cortex, as well as temporoparietal regions, the amygdala and anterior cingulate, yet again suggesting region-specific effects in frontolimbic brain areas that are also involved in the processing of affective states and $\operatorname{mood}[5,36,40,52,56]$. Hence, stress reduction, e.g., through exercise, has been shown to involve serotoninergic and especially dopaminergic and neuropeptidergic signalling in the associated brain regions $[5,33,52]$. Dopamine, for example, seems to be of particular interest here for spatial memory, concentration and positive mood, as well as for the general effectiveness of the brain performance, or the reestablishment and safekeeping of a normal brain function in the course, e.g., of exhausting (cognitive) tasks [33]. Thus, dopamine-enhancing exercises and activities appear to be suitable means to balance stress on the neurobiological level [5]. Moreover, these various signalling molecules have to be finely tuned in challenging tasks, especially when prolonged or enduring.

The chronology and sequence of stress and stress-reducing hormone or neurotransmitter release during exercise not only depends on the actual point in time of the release, but also on the concentration and half-life, i.e., kinetic pattern, of the hormone into question. For example, BDNF and cortisol differ in that they both are produced and elevated through rampant or strenuous and exhausting exercise, however, BDNF returns to base levels instantly after the exercise challenge is over, while cortisol recovers slower [60]. Furthermore, the type of exercise is essential, as noted before, with aerobic and moderate exercise being optimally suited, in fact crucial, for molecular stress reduction and health [5]. Long-term exercise, such as distant running, can lead to an euphoric state that is sometimes called 'runner's high' [5], enabling the runner to proceed with the task, though exhausting. The neurochemical correlates of this exerciseinduced positive mood change critically build on opioidergic mechanisms in the brain, that is, opioid receptor activation, preferentially in prefrontal and limbic and paralimbic brain structures $[5,56]$. The runner's high phenomenon is an obvious result of autoregulatory opioid signalling [5]. The opioid peptides $\beta$-endorphin and Met-enkephalin with its precursor proenkephalin originate from the anterior pituitary, where proopiomelanocortin (POMC) is produced, again showing the connection between this signalling system and the central stress axes, as illustrated. Interestingly, prolactin is also enhanced during and post-exercise, comparable to cortisol, however, this latter hormone is a partial dopamine inhibitor that comes consecutively (i.e., delayed) after dopamine release in the stressexercise sequence was initiated [61], comparable to the endorphins [5]. Prolactin also originates from the anterior pituitary. The more prolactin builds up in an exercise, e.g., sexual activity, the deeper the relaxation and satisfaction later [61], comparable to the postulated dopamine-morphine sequence [5]. In other words, the greater the stress (in combination with physical activity and exhaustion), the deeper the relief and relaxation, i.e., endogenous stress reduction and reward (the "I did 
it' component), when successfully solving and overcoming the stressful challenge [5].

Finally, since dopamine, endocannabinoids and morphine (and even serotonin) exert their effects, in part, via NO release, it is not surprising to find substantial constitutive NO activity in exercise [5, $43,58,59,62]$. We surmise that this potentially protective neurobiological signalling molecule plays the role of an effector for many of the observed phenomena of autoregulation [5, 58]. For example, positive results with exercise therapy in stroke patients are abolished by the inhibition of NO signalling [43]. Moreover, NO counteracts NE, that is, cellular stress, on the neuronal level, thereby explaining a self-regulatory anti-stress capacity of NO-enhancing activities [5, 6, 57, 58].

Taken together, we hypothesize that exercise induces autoregulatory stress reduction via limbic pleasure and reward pathways, using, at least in parts, the same neurobiological components like other SM tools. Positive activities and cognitive behaviors ('thoughts') as well as moderate physical exercises neurobiologically project on the hypothalamus and the pituitary gland, i.e., the central stress axes, via prefrontal/frontolimbic pathways, thereby inducing a central vegetative stress reduction, making it possible to mentally (i.e., self-regulatory, deliberately) influence stress and autonomous functions throughout the body, e.g., mind-body medicine $[48,49]$. Stress hormones, including NE, cortisol and corticotropin-releasing hormone $(\mathrm{CRH})$, get reduced, or their effects antagonized on the receptor level, while dopamine, opioids and opiates are endogenously enhanced, that is, their signalling systems initiated [5]. Serotonin and dopamine improve physical endurance capacities and stress hardiness, as do the endorphins, subsequently, with endogenous morphine presumably playing a role in the recovery phase of stress and/or strenuous exercise [5]. The latter substances also improve mood and pain resistance, two critical features of a successful stress autoregulation [47]. All these effects are potentially plastic, that is, learnable and modifiable. However, research in this area has just begun to reveal the underlying potential. The basic science and methodology is complex: this may be due to the fact that measuring NO in vivo (i.e., real-time), for example, is technically challenging, and additionally, human brain tissue for morphine detection is not easy to obtain, and non-invasive (particularly: molecular) brain imaging determinations are still expensive [63-66]. Finally, the effects in question, though principally measurable, are sometimes rather subtle and, at times, difficult to interpret, or even unexpected, and thus some research still waits for confirmation by independent research groups $[5,6,67,68]$.

\section{Conclusions}

Stress is a problem of our time. This is not only true for clinical or medical settings, but also for the work environment. Stress can facilitate illness ('disease') and is responsible for a surge in medical spending for the treatment of, e.g., cardiovascular diseases, as well as for lower profits and higher absenteeism rates in the business world. Thus, besides clinical and external sources of stress and reasons for it, particularly psychosocial and their treatment, the endogenous or behavioral reduction and therapy of stress is still much-needed.

Research has suggested different ways and approaches to self-treat stress or buffer against stressors and their impacts. These self-healing or self-care-centred approaches are sometimes referred to as mind-body medicine or stress management strategies. They consist of various cognitive behavioral techniques, as well as relaxation exercises and nutritional counselling or therapy. However, a central part of all modern stress management programs are exercise practices.

At the core, all these approaches combine different elements to reduce stress or learn to cope with it, therefore relying on endogenous resources for health and resistance (i.e., resilience). With regard to the brain and underlying neurobiological mechanisms and pathways, endogenous reward and motivation circuitries that are imbedded in the limbic regions of the brain are responsible for the autoregulatory and endogenous reduction of stress. Exercise techniques clearly have an impact upon these systems. This is one of the reasons why physical activity not only improves overall physical health and strength, or physiologically reduces stress, but also increases mood and decreases psychological distress, i.e., depression or affective instability, by pleasure induction.

Our brain-dependent autoregulation critically relies on an orchestra of effective - and subjectively rewarding - signalling molecules to alter stress, e.g., endogenous morphine and coupled NO pathways. In combination with the pleasurable experience of a rewarding activity, which is additionally associated, for example, with the release of dopamine in the initial phase, these stress-altering actions and coupled neurobiological mechanisms represent a finely tuned autoregulatory 'slope', that is, an endogenous potential for stress reduction and improvement of health and facilitation of prevention. Evolutionarily and biologically, the various activities and pathways, as depicted in this work, are linked together, which can also be demonstrated by the fact that dopamine is endogenously and, by enzymatic processing, converted into morphine. Additionally, morphine signalling leads to enhanced NO release by the constitutive NO synthases. 


\section{References}

1. Badura B, Schroeder H, Klose J, Macco K (eds.). Absenteeism Report 2009: Work and psyche - reducing strain, enhancing well-being [German]. Springer, Berlin 2009.

2. Siegrist J. Chronic psychosocial stress at work and risk of depression: evidence from prospective studies. Eur Arch Psychiatry Clin Neurosci 2008; 258 Suppl 5: 115-9.

3. Salavecz G, Chandola T, Pikhart H, et al. Work stress and health in Western European and post-communist countries: An East-West comparison study. J Epidemiol Community Health 2010; 64: 57-62.

4. Esch T. Health in Stress: Change in the Stress Concept and its Significance for Prevention, Health and Life Style [German]. Gesundheitswesen 2002; 64: 73-81.

5. Esch T, Stefano GB. The neurobiology of stress management. Neuro Endocrinol Lett 2010; 31: 19-39.

6. Stefano GB, Benson H, Fricchione GL, Esch T (eds.). The stress response: always good and when it is bad. Medical Science International, Warsaw-New York 2005.

7. Esch T, Stefano GB, Fricchione GL. The therapeutic use of the relaxation response in stress-related diseases. Med Sci Monit 2003; 9: RA23-34.

8. Cannon W. The emergency function of the adrenal medulla in pain and the major emotions. Am J Physiol 1914; 33: 356-72.

9. Cannon WB. Bodily changes in pain, hunger, fear, and rage; an account of recent researchers into the function of emotional excitement. New York: Appleton and Company; 1915.

10. Esch T, Stefano GB, Fricchione GL, Benson H. An overview of stress and its impact in immunological diseases. Mod Asp Immunobiol 2002; 2: 187-92.

11. Esch T, Stefano GB, Fricchione GL, Benson H. Stress in cardiovascular diseases. Med Sci Monit 2002; 8: RA93-101.

12. Esch T, Stefano GB, Fricchione GL, Benson $\mathrm{H}$. The role of stress in neurodegenerative diseases and mental disorders. Neuroendocrinol Lett 2002; 23: 199-208.

13. Sterling P, Eyer J. Allostasis: a new paradigm to explain arousal pathology. In: Fisher S, Reason J (eds). Handbook of life stress, cognition and health. New York: John Wiley \& Sons; 1988.

14. McEwen BS. Protective and damaging effects of stress mediators. N Engl J Med 1998; 338: 171-9.

15. Negrao AB, Deuster PA, Gold PW, Singh A, Chrousos GP. Individual reactivity and physiology of the stress response. Biomed Pharmacother 2000; 54: 122-8.

16. Kream R, Stefano GB. Endogenous morphine and nitric oxide coupled regulation of mitochondrial processes. Med Sci Monit 2009; 15: RA263-8.

17. Stefano GB, Kream RM, Esch T. Revisiting tolerance from the endogenous morphine perspective. Med Sci Monit 2009; 15: RA189-98.

18. Stefano GB, Esch T, Kream R. Xenobiotic perturbation of endogenous morphine signalling: paradoxical opiate hyperalgesia. Med Sci Monit 2009; 15: RA107-10.

19. Stefano GB, Stefano J, Esch T. Anticipatory stress response: a significant commonality in stress, relaxation, pleasure and love responses. Med Sci Monit 2008; 14: RA17-21.

20. Esch T, Stefano GB. The neurobiology of pleasure, reward processes, addiction and their health implications. Neuroendocrinol Lett 2004; 25: 235-51.

21. Bozarth MA. Pleasure systems in the brain. In: Wartburton DM (ed.). Pleasure: the politics and the reality. New York: Wiley \& Sons, 1994

22. Bob P, Fedor-Freybergh P, Jasova D, et al. Dissociative symptoms and neuroendocrine dysregulation in depression. Med Sci Monit 2008; 14: CR499-504.
23. Troland LT. The fundamentals of human motivation. New York: Van Nostrand Reinhold, 1928.

24. Mravec B, Ondicova K, Valaskova Z, Gidron Y, Hulin I. Neurobiological principles in the etiopathogenesis of disease: when diseases have a head. Med Sci Monit 2009; 15: RA6-16.

25. Esch T, Guarna M, Bianchi E, Zhu W, Stefano GB. Commonalities in the central nervous system's involvement with complementary medical therapies: limbic morphinergic processes. Med Sci Monit 2004; 10: MS6-17.

26. Nestler EJ. Molecular basis of long-term plasticity underlying addiction. Nat Rev Neurosci 2001; 2: 119-28.

27. Nestler EJ, Malenka RC, Hyman SE. Molecular basis of neuropharmacology. Columbus: McGraw-Hill, 2001.

28. Fricchione G, Zhu W, Cadet $P$, et al. Identification of endogenous morphine and a micro3-like opiate alkaloid receptor in human brain tissue taken from a patient with intractable complex partial epilepsy. Med Sci Monit 2008; 14: CS45-9.

29. Bozarth MA. Ventral tegmental reward system. In: Oreland L, Engel J (eds.). Brain Reward Systems and Abuse. New York: Raven Press 1987.

30. Metz U, Welke J, Esch T, Renneberg B, Braun V, Heintze C. Perception of stress and quality of life in overweight and obese people - implications for preventive consultancies in primary care. Med Sci Monit 2009; 15: PH1-6.

31. Hillman CH, Erickson KI, Kramer AF. Be smart, exercise your heart: exercise effects on brain and cognition. Nat Rev Neurosci 2008; 9: 58-65.

32. Voelcker-Rehage C, Godde B, Staudinger UM. Activity, physical and psychological mobility in old age. Bundesgesundheitsblatt Gesundheitsforschung Gesundheitsschutz 2006; 49: 558-66.

33. Stroth S, Hille K, Spitzer M, Reinhardt R. Aerobic endurance exercise benefits memory and affect in young adults. Neuropsychol Rehabil 2009; 19: 223-43.

34. Herholz K, Buskies W, Rist M, Pawlik G, Hollmann W, Heiss WD. Regional cerebral blood flow in man at rest and during exercise. J Neurol 1987; 234: 9-13.

35. Hollman W, Strueder HK. Exercise, physical activity, nutrition, and the brain. Nutr Rev 1996; 54: 537-43.

36. Hollmann W, Strueder HK. Brain function, mind, mood, nutrition, and physical exercise. Nutrition 2000; 16: 516-9.

37. Hollmann W, Strueder HK. Brain, psyche and physical activity. Orthopade 2000; 29: 948-56.

38. Ben-Sefer E, Ben-Natan M, Ehrenfeld M. Childhood obesity: current literature, policy and implications for practice. Int Nurs Rev 2009; 56: 166-73.

39. van Praag H, Shubert T, Zhao C, Gage FH. Exercise enhances learning and hippocampal neurogenesis in aged mice. J Neurosci 2005; 25: 8680-5.

40. Erickson K, Kramer AF. Aerobic exercise effects on cognitive and neural plasticity in older adults. Br J Sports Med 2009; 43: 22-4.

41. Lazar SW, Kerr CE, Wasserman RH, et al. Meditation experience is associated with increased cortical thickness. Neuroreport 2005; 16: 1893-7.

42. Wolf SL, Winstein CJ, Miller JP, et al. Effect of constraintinduced movement therapy on upper extremity function 3 to 9 months after stroke: the EXCITE randomized clinical trial. JAMA 2006; 296: 2095-104

43. Gertz K, Priller J, Kronenberg G, et al. Physical activity improves long-term stroke outcome via endothelial nitric oxide synthase-dependent augmentation of neovascularization and cerebral blood flow. Circ Res 2006; 99: 1132-40. 
44. Esch T. Stress, adaptation, and self-organization: Balancing processes facilitate health and survival [German]. Forsch Komplementarmed Klass Naturheilkd 2003; 10: 330-41.

45. Ernst S, Welke J, Heintze C, et al. Effects of mindfulnessbased stress reduction on quality of life in nursing home residents: A feasibility study. Forsch Komplementmed 2008; 15: 74-81.

46. Ernst S, Esch SM, Esch T. The impact of mindfulnessbased interventions on health care [German]. Forsch Komplementmed 2009; 16: 296-303.

47. Esch T, Stefano GB. A bio-psycho-socio-molecular approach to pain and stress management. Forsch Komplementmed 2007; 14: 224-34.

48. Esch T, Duckstein J, Welke J, Braun V. Mind/body techniques for physiological and psychological stress reduction: Stress management via Tai Chi training - a pilot study. Med Sci Monit 2007; 13: CR488-97.

49. Esch T. Mind-Body Medicine: stress, stress management and health promotion. Kompl Integr Med 2008; 49: 35-9.

50. van Donkelaar EL, van den Hove DL, Blokland A, Steinbusch HW, Prickaerts J. Stress-mediated decreases in brain-derived neurotrophic factor as potential confounding factor for acute tryptophan depletioninduced neurochemical effects. Eur Neuropsychopharmacol 2009; in press.

51. van Praag H. Neurogenesis and exercise: past and future directions. Neuromolecular Med 2008; 10: 128-40.

52. Pollak DD, Monje FJ, Zuckerman L, Denny CA, Drew MR, Kandel ER. An animal model of a behavioral intervention for depression. Neuron 2008; 60: 149-61.

53. Guarna M, Ghelardini C, Galeotti N, et al. Effects of endogenous morphine deprivation on memory retention of passive avoidance learning in mice. Int J Neuropsychopharmacol 2004; 7: 311-9.

54. McEwen BS. Plasticity of the hippocampus: adaptation to chronic stress and allostatic load. Ann NY Acad Sci 2001; 933: 265-77.

55. Kucukatay V, Hacioglu G, Ozkaya G, Agar A, Yargicoglu P. The effect of diabetes mellitus on active avoidance learning in rats: the role of nitric oxide. Med Sci Monit 2009; 15: BR88-93.

56. Boecker H, Sprenger T, Spilker ME, et al. The runner's high: opioidergic mechanisms in the human brain. Cereb Cortex 2008; 18: 2523-31.

57. Esch T, Stefano GB. Proinflammation: a common denominator or initiator of different pathophysiological disease processes. Med Sci Monit 2002; 8: HY1-9.

58. Esch T, Stefano GB, Fricchione GL, Benson H. Stress-related diseases: a potential role for nitric oxide. Med Sci Monit 2002; 8: RA103-18.

59. Stefano GB, Prevot V, Cadet P, Dardik I. Vascular pulsations stimulating nitric oxide release during cyclic exercise may benefit health: a molecular approach (review). Int J Mol Med 2001; 7: 119-29.

60. Rojas Vega S, Strueder HK, Wahrmann BV, Schmidt A, Bloch W, Hollmann W. Acute BDNF and cortisol response to low intensity exercise and following ramp incremental exercise to exhaustion in humans. Brain Res 2006; 1121: 59-65.

61. Rojas Vega S, Strueder HK, Wahrmann BV, Bloch W, Hollmann W. Bicarbonate reduces serum prolactin increase induced by exercise to exhaustion. Med Sci Sports Exerc 2006; 38: 675-80.

62. Mantione KJ, Esch T, Stefano GB. Detection of nitric oxide in exhaled human breath: Exercise and resting determinations. Med Sci Monit 2007; 13: MT1-5.

63. Atmanene C, Laux A, Glattard E, et al. Characterization of human and bovine phosphatidylethanolamine-binding protein (PEBP/RKIP) interactions with morphine and morphine-glucuronides determined by noncovalent mass spectrometry. Med Sci Monit 2009; 15: BR178-87

64. De Gennaro Colonna V, Bianchi M, Pascale V, et al. Asymmetric dimethylarginine (ADMA): An endogenous inhibitor of nitric oxide synthase and a novel cardiovascular risk molecule. Med Sci Monit 2009; 15: RA91-101.

65. Santamaria F, De Stefano S, Montella S, et al. Nasal nitric oxide assessment in primary ciliary dyskinesia using aspiration, exhalation, and humming. Med Sci Monit 2008; 14: CR80-5.

66. Cable D, Celotto A, Evora PR, Schaff H. Asymmetric dimethylarginine endogenous inhibition of nitric oxide synthase causes differential vasculature effects. Med Sci Monit 2009; 15: BR248-53.

67. Misra M, Sarwat M, Bhakuni P, Tuteja R, Tuteja N. Oxidative stress and ischemic myocardial syndromes. Med Sci Monit 2009; 15: RA209-19.

68. Erken G, Bor Kucukatay M, Erken H, Kursunluoglu R, Genc O. Influence of classical and rock music on red blood cell rheological properties in rats. Med Sci Monit 2008; 14: BR28-33. 